\title{
Prospective study of the radiological changes in hands, feet, and cervical spine in adult rheumatoid disease
}

\author{
JOHN WINFIELD, ADAM YOUNG, PETER WILLIAMS, AND MARY \\ COR B E T T
}

From the Department of Rheumatology, the Middlesex Hospital, Arthur Stanley House, Tottenham Street, London W1P 9PG

SUMMARY Annual radiographs of hands, feet, and cervical spine were taken in 100 patients with rheumatoid arthritis from the first year of disease for a mean follow-up period of 9.5 years. Seventy-six patients developed peripheral erosive disease and 54 developed rheumatoid changes of the cervical spine, of whom $34(63 \%)$ had subluxations. The severity of rheumatoid neck damage correlated strongly with the severity of peripheral erosive disease $(p=0.002)$. Cervical subluxation was more likely to occur in patients with erosions of the hands and feet which deteriorated progressively with time $(\mathrm{p}=0 \cdot 018)$. The timing and severity of cervical subluxation coincided with the progression of peripheral erosive disease in 26 of these 34 patients $(76 \cdot 5 \%)$. The other 8 patients with cervical subluxation (23.5\%) had none or only mild peripheral erosions, but their subluxations did not progress with time. There were 9 patients with marked cervical subluxations which deteriorated relentlessly, and they all also had severe progressive erosive disease of the hands and feet. One of these patients developed a cervical myelopathy, and 2 other patients with normal neurological signs had upper cervical fusions performed for severe occipital headache. This small group of rheumatoid patients who are at risk of developing cervical myelopathy cannot be predicted with certainty, but can be selected out at an early stage by performing regular radiographs of hands, feet, and cervical spine.

The prospective study of patients with early rhe umatoid arthritis (RA) at the Middlesex Hospital has been in progress since 1966 . Only patients with RA of less than one year have been included, and regular follow-up has been maintained now in over 100 patients for up to 15 years.

Brook and Corbett ${ }^{1}$ reported on the changes in radiographs of the hands and feet in the first 94 patients in this prospective study and found that $\mathbf{7 1 . 3 \%}$ developed erosions over a mean follow-up period of 63.1 months. Nearly $90 \%$ of these patients developed their erosions within 2 years of entry to the study, and a subgroup of erosive patients was identified who became radiologically static within the first few years of their disease. Also reporting on this prospective study, Winfield et al. ${ }^{2}$ have clarified the natural history of the radiological changes of the cervical spine in RA by showing that when cervical

Accepted for publication 6 December 1982.

Correspondence to Dr J. Winfield. subluxation occurs it is similarly usually an early feature of the disease.

In this report we have reviewed the yearly radiographs both of the hands and feet and of the cervical spines in 100 patients with RA. This was undertaken to see if the peripheral and cervical $x$-ray changes coincided in timing and severity, and if it was possible to identify those patients at risk of developing cervical myelopathy early in the course of their disease.

\section{Patients and methods}

The selection criteria for patients and the organisation of the Middlesex Hospital prospective study have been previously described by Fleming et al. ${ }^{3} \mathrm{At}$ entry to the study all patients had RA of less than one year's duration. Thirty-seven patients fulfilled the 'probable' or 'possible' criteria for RA according to the American Rheumatism Association (ARA), ${ }^{4}$ and 63 fulfilled the 'definite' or 'classical' criteria within 
the first year. Functional grade was assessed according to Steinbrocker et al. ${ }^{5}$ There were 43 males and 57 females, with a mean age at presentation of 47.6 years. The mean follow-up period was 9.5 years, ranging from 4 to 15 years, and all patients are still attending.

The radiographs of hands, feet, and cervical spine were taken annually and each patient's series of films studied to assess the presence, timing, and severity of rheumatoid damage. The criteria for the presence of bony erosions in the radiographs of the hands and feet were those reported by Brook ${ }^{\mathbf{1}}$ and graded into none, mild, moderate, and severe erosive changes as described by Lawrence. ${ }^{6}$ These standards have been shown to give consistent reproducible readings with a low intraobserver error except when the radiographic appearances are minimal. This latter problem was partially overcome because of the availability of annual sequential films, which allowed us to assess the development and progression of peripheral erosions over a number of years.

The criteria used for the presence of rheumatoid changes in the cervical spine were those modified from the study of Bland ${ }^{7}$ and described in detail by Winfield et al. ${ }^{2}$

The radiographs of hands and feet were read independently by one author (A.Y.) and the radiographs of necks read by J.W., again independently and without knowledge of the patient's clinical details. The radiographs of 20 of these patients were read independently by a third rheumatologist (P.W.), and the findings concurred with the original radiological classification except in 2 patients, in whom the distinction between mild and moderate $x$-ray changes was borderline.

\section{Results}

Comparison of the severity of rheumatoid changes in hands and feet with cervical spines

The severity of peripheral RA as assessed on the latest radiographs of the hands and feet correlated strongly with the severity of cervical spine changes $\left(\chi^{2}=25 \cdot 7, p=0 \cdot 0022\right.$, Table 1$)$. This association was still present when the presence and absence of all rheumatoid features in the cervical spines were compared with the presence and absence of bony erosions in the hands and feet $\left(\chi^{2}=5 \cdot 4, p=0.01\right)$.

To overcome the problem of possible subjectivity of the radiological assessment into mild, moderate, and severe changes the sequential $x$-rays of the hands and feet were divided into 3 broad groups according to the presence or absence of erosive disease, and whether this progressed or not after 2 years of study. Fifty-five patients developed peripheral erosions which progressed relentlessly after 2 years of study or displayed a series of progressive and static phases. In
Table 1 Comparison of the severity in $\mathrm{x}$-rays of hands and feet with severity in those of cervical spine

\begin{tabular}{lccccc}
\hline $\begin{array}{l}\text { Cervical } \\
\text { spine }\end{array}$ & \multicolumn{6}{l}{$X$-rays of hands and feet } \\
\cline { 2 - 6 } & Total & None & Mild & Moderate & Severe \\
\hline None & 46 & 16 & 9 & 13 & 8 \\
Mild & 28 & 4 & 9 & 10 & 5 \\
Moderate & 22 & 4 & 7 & 9 & 2 \\
Severe & 4 & 0 & 0 & 2 & 2 \\
Total & 100 & 24 & 25 & 34 & 17 \\
\hline
\end{tabular}

$\chi^{2}=25 \cdot 7, \mathrm{p}=0.0022$.

21 patients with peripheral erosive disease the erosions did not progress with time. There were 24 patients with nonerosive disease throughout the whole follow-up period.

In addition the radiographs of the cervical spines were also divided into groups according to the presence and type of or absence of rheumatoid neck damage. Fifty-four patients had rheumatoid changes in serial radiographs of the cervical spine. Thirty-four patients developed cervical subluxations; 5 with atlantoaxial, 24 with subaxial subluxations, and 5 with both. Twenty had other rheumatoid neck changes which included vertebral end plate erosions and disc space narrowing of the upper cervical spine. Forty had degenerative changes only, and 6 patients had no abnormalities on their serial cervical spine radiographs. Of the patients with cervical RA changes 25 progressed with time, whereas 29 remained static after the first 2 years of follow-up.

Comparison of cervical spine changes with progression of peripheral $x$-rays

Of the 55 patients with progressive peripheral erosive disease $39(70.9 \%)$ developed rheumatoid changes in the cervical spine at some time, in comparison with 7 of $21(33.3 \%)$ patients whose erosions did not progress with time and 8 of $24(33.3 \%)$ without peripheral erosions (Table 2). Twenty-six patients developed cervical subluxation and 13 developed other RA changes ( $49 \%$ and $23.6 \%$ of the progressive peripheral erosive group respectively). All 9 patients who had severe subluxations which deteriorated with time also had marked progressive peripheral erosive disease.

Fig. 1 shows the cervical spine $x$-rays of a 52-yearold woman who in 1968 developed RA which was difficult to control despite the vigorous use of disease-modifying drugs. By 1972 there was significant atlanto-axial subluxation which progressed to an $11 \mathrm{~mm}$ displacement by 1981 . She also had marked progressive erosive changes in the radiographs of hands and feet. She complained of severe occipital 

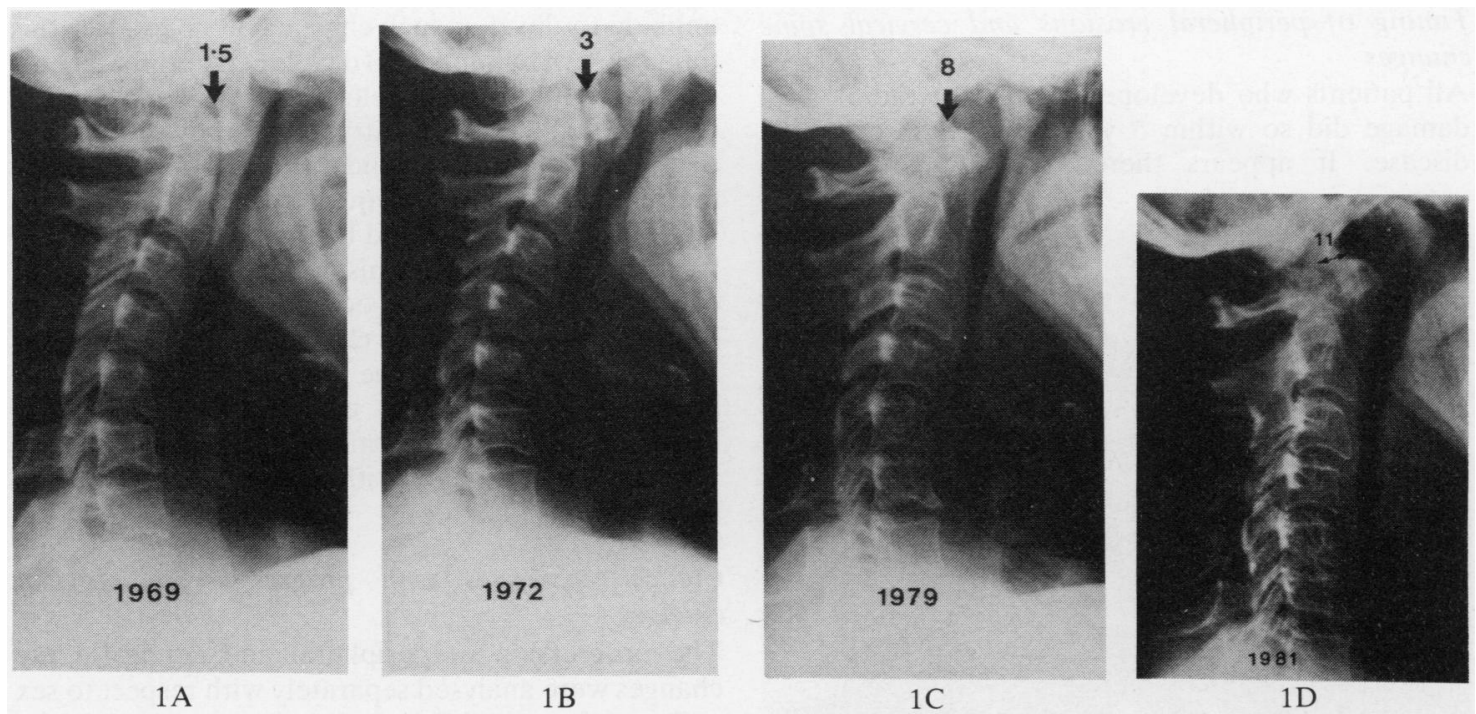

Fig. 1 Lateral cervical spine radiographs of a female with $R A$ and severe progressive peripheral erosive disease showing a relentless deterioration in cervical subluxation from 1968 to 1981 . By 1981 atlanto-axial subluxation of $11 \mathrm{~mm}$ in full flexion had occurred.

Table 2 Comparison of radiographs of hands and feet with those of cervical spine

\begin{tabular}{|c|c|c|c|c|}
\hline \multirow{3}{*}{$\begin{array}{l}X \text {-rays of } \\
\text { cervical spines }\end{array}$} & & \multicolumn{3}{|c|}{$X$-rays of hands and feet } \\
\hline & & \multicolumn{2}{|l|}{ Erosive } & \multirow[t]{2}{*}{ Nonerosive } \\
\hline & & Progressive & Static & \\
\hline Total & 100 & 55 & 21 & 24 \\
\hline Subluxation & 34 & 26 & 4 & 4 \\
\hline Other RA changes & 20 & 13 & 3 & 4 \\
\hline OA changes only & 40 & 15 & 13 & 12 \\
\hline Normal & 6 & 1 & 1 & 4 \\
\hline
\end{tabular}

A strong correlation was found between the presence or absence of cervical spine changes and progressive or static crosions of the hands and feet $\left(\chi^{2}=14 \cdot 6, p=0 \cdot 001\right)$.

When the analysis was restricted to patients with cervical spine changes of RA only, a weaker association was found with progressive or static peripheral erosions $\left(\chi^{2}=7 \cdot 9, p=0 \cdot 018\right)$.

headaches and neck pain resulting in marked restriction of neck mobility, but neurological examination was normal. In 1981 she had a posterior C1-2 fusion with wire and a small bone graft.

One further patient, a 48-year-old Indian whose peripheral erosions showed a progressive deterioration, developed a mild subaxial subluxation at C2-3 within 2 years. After 4 years of study he also developed a progressive atlanto-axial subluxation. $\mathrm{He}$ had no neurological signs but complained of severe occipital headache. A successful upper cervi- cal fusion was performed, the principal indication being occipital pain.

Patients with progressive peripheral erosive disease developed more rheumatoid neck changes such as disc space narrowing in the upper cervical spine and erosions of the vertebral plates as well as atlanto-axial and subaxial subluxation. This association was significant both in severity and in timing $(\mathrm{p}=$ $0.001)$. It should also be noted, however, that 4 patients without peripheral erosive disease and 4 with erosive but nonprogressive radiographs of hands and feet also developed cervical subluxation (Table 2 ). The subluxations in these 8 patients were mild and did not deteriorate and are probably not clinically significant.

For example, Fig. 2 shows the lateral cervical spine $x$-ray of a 54-year-old woman with 'probable' RA for 7 years. She had gained a long-lasting remission after treatment with gold in the first 2 years of her disease. The radiographs of her hands and feet did not show erosive disease but she developed subaxial subluxation which remained static.

Two other patients also in this group developed atlanto-axial subluxation greater than $2.5 \mathrm{~mm}$. There were no special features of the 16 patients who had progressively worsening erosions of the hands and feet but who had only degenerative changes or normal radiographs of cervical spines. Clearly further follow-up of these patients is essential to establish the eventual outcome. 
Timing of peripheral erosions and cervical spine changes

All patients who developed significant radiological damage did so within 5 years of the onset of the disease. It appears therefore that patients are

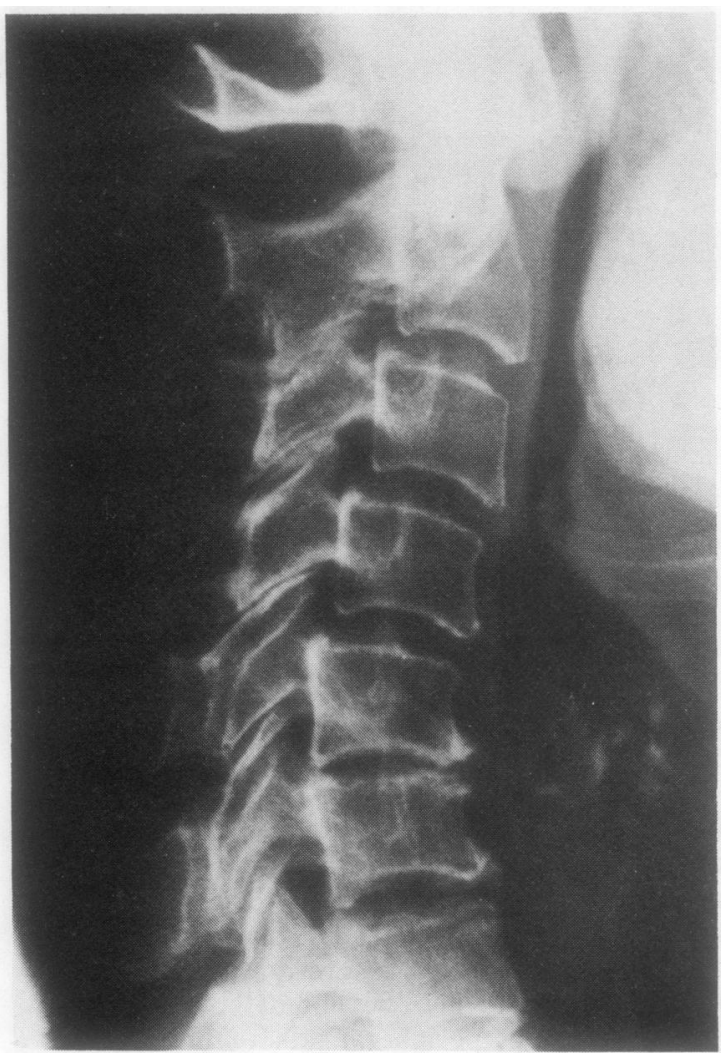

Fig. 2 Lateral cervical spine radiograph of 54-year-old woman with 'probable' RA for 7 years showing multiple subaxial slips. She had seropositive but nonerosive peripheral disease. unlikely to develop such changes de novo after this time. $86 \%$ of patients who developed peripheral erosions and $80 \%$ who developed cervical subluxation did so within 2 years of entry to the study. In addition the rate of deterioration and the timing of progressive and static phases in the sequential films of hands and feet were closely mirrored by the progression of cervical subluxation when this occurred. Deterioration of subluxation with time occured only in 9 patients (e.g., Fig. 1), and in 3 the clinical features warranted surgical intervention. The other 6 patients in this group did not show any evidence of neurological deficit at the last assessment. The disease in the remaining 17 patients with subluxations remained static.

Clinical correlates with progressive radiological changes

The progression of peripheral and cervical $x$-ray changes were analysed separately with respect to sex and age of onset of RA, and to the recordings in the first year of study of the presence of rheumatoid factor and ARA criteria, and their functional capacity recorded at the last visit (Table 3).

\section{Radiographs of hands and feet}

The age of onset of RA was slightly younger in the worse radiological group, but there was no significant difference between mean age of onset or sex and severity of $x$-rays, and the duration of disease and mean follow-up were similar in all groups (Table 3). As might be expected, a higher incidence of seropositivity in the first year of study was found in patients with peripheral erosive disease compared with nonerosive RA $\left(\chi^{2}=13 \cdot 6, p=0.0004\right)$, and also in those with progressive erosive disease compared to static but erosive $\mathrm{RA}\left(\chi^{2}=5 \cdot 5, \mathrm{p}=0.018\right)$. More ARA criteria in the first year of study were found in patients with erosive disease $\left(\chi^{2}=48 \cdot 6, p=\right.$ $0 \cdot 00001)$, but there was no significant difference

Table 3 Clinical correlates with progressive radiological changes

\begin{tabular}{|c|c|c|c|c|c|c|c|c|c|c|c|c|c|}
\hline & \multirow[t]{2}{*}{ No. } & \multirow[t]{2}{*}{$M: F$} & \multirow{2}{*}{$\begin{array}{l}\text { Mean } \\
\text { age } \\
\text { (onset) }\end{array}$} & \multicolumn{3}{|l|}{$R F$} & \multicolumn{4}{|c|}{ ARA grade } & \multicolumn{3}{|c|}{ Functional grade } \\
\hline & & & & + & \pm & - & 1 & 2 & 3 & 4 & 1 & 2 & 3 \\
\hline \multicolumn{14}{|c|}{ Radiographs of hands and feet } \\
\hline Progressive & 55 & $21: 34$ & $45 \cdot 7$ & 47 & 5 & 3 & 0 & 10 & 24 & 21 & 18 & 30 & 7 \\
\hline Static & 21 & $11: 10$ & $46 \cdot 2$ & 16 & 1 & 4 & 0 & 6 & 11 & 4 & 16 & 4 & 1 \\
\hline Nonerosive & 24 & $11: 13$ & $50 \cdot 9$ & 9 & 5 & 10 & 10 & 11 & 2 & 1 & 19 & 5 & 0 \\
\hline \multicolumn{14}{|c|}{ Radiographs of cervical spine } \\
\hline Progressive & 25 & $8: 17$ & $51 \cdot 1$ & 20 & 1 & 4 & 1 & 3 & 13 & 8 & 12 & 9 & 4 \\
\hline Static & 29 & $13: 16$ & $45 \cdot 8$ & 26 & 1 & 2 & 3 & 6 & 10 & 10 & 15 & 10 & 4 \\
\hline Non-RA & 46 & $22: 24$ & $46 \cdot 1$ & 26 & 9 & 11 & 6 & 18 & 14 & 8 & 26 & 20 & 0 \\
\hline Total & 100 & $43: 57$ & $47 \cdot 6$ & 72 & 11 & 17 & 10 & 27 & 37 & 26 & 53 & 39 & 8 \\
\hline
\end{tabular}

$\mathbf{R F}=$ rheumatoid factor. 
when progressive erosive disease was compared with nonprogressive disease. Worse functional grades at the latest visit were found in the erosive group $\left(\chi^{2}=\right.$ $8 \cdot 6, p=0.003)$ and the progressive erosive group $\left(\chi^{2}\right.$ $=11 \cdot 6, \mathrm{p}=0 \cdot 001)($ Table 3$)$.

\section{Radiographs of cervical spine}

The mean age of onset of RA was slightly older (but not statistically significantly so) in patients with progressive radiological changes of the cervical spine (Table 3). A higher proportion of females than expected had progressive changes in the cervical spines (68\%), particularly those with severe subluxation (66\%). More ARA criteria in the first year and worse functional capacity were associated with rheumatoid changes in the cervical spine (respectively $\chi^{2}=8 \cdot 5, p=0.03$; and $\left(\chi^{2}=7 \cdot 4, p=0 \cdot 02\right)$ but not with the development of progressive disease. The presence of rheumatoid factor alone was not significantly related to cervical spine changes, but highly seropositive patients were more likely to develop subluxations $\left(\chi^{2}=22 \cdot 1, p=0 \cdot 008\right)$, whether progressive or not.

\section{Discussion}

The prognostic value of performing yearly radiographs of both the hands and the feet in the early stages of RA has already been demonstrated in reports on patients in this prospective study. ${ }^{1}$ The severity of peripheral bony erosions in the early years of RA has been shown to correlate both with the degree of inflammatory joint activity when assessed clinically ${ }^{8}$ and with the eventual clinical outcome. ${ }^{9}$ It follows therefore that disease activity should be carefully evaluated in the early years of the disease and suppressive therapy used before significant joint damage has occurred.

The present study has clearly shown that peripheral joint erosions and cervical subluxation tend to coincide in both severity and timing, suggesting that these processes are closely linked to the current disease activity. Sharpet al. ${ }^{10}$ were among the first to note the increased frequency of radiological damage in the cervical spine with damage to the hands and feet in a retrospective study of RA. The only other report on this subject from long-term prospective studies in RA comes from Rasker and Cosh. ${ }^{11}$ They followed up 62 patients for 15 years and found a significant correlation between the radiological damage of the metacarpophalangeal joints and the cervical spine, and also suggested that steroid medication had an independent deleterious effect on cervical spine changes. The patients in these studies were not $x$-rayed as frequently as those of this study, so that early details of radiographic changes are mis- sing. Too few of our patients had received steroids for firm conclusions to be drawn, though a similar trend was found.

The development of cervical subluxation is more likely in patients with erosions of the hands and feet, especially if these progress, but even then the majority of subluxations do not deteriorate with time. The reason for this is uncertain, but bony or fibrous ankylosis after the inflammatory component has resolved may be a factor in stabilising affected segments.

In 8 of our patients the radiological changes of RA were confined to the cervical spine, and all these patients had both mild clinical and radiological disease. This may reflect the known heterogeneity of RA but also suggests that the values of 2.5 or $3 \mathrm{~mm}$ of subluxation which are accepted as representing atlanto-axial instability might be too conservative, and the higher value of $4 \mathrm{~mm}$ would be more practical.

Cervical radiculopathy and myelopathy, developing as a result of erosive disease of the cervical spine, although fortunately not common, is known to be a troublesome and severe complication of RA. In this series $34 \%$ developed atlanto-axial or subaxial subluxation, but in only 9 patients did the subluxations progress after 2 years, and all had severe erosive changes of the hands and feet. One of these developed a myelopathy, and 2 others have had upper cervical fusions to alleviate occipital pain and to prevent a myelopathy developing. We have not yet observed severe progressive subaxial subluxation in our patients. This may be a more important contributory cause of myopathy at a later stage of the disease.

Only a small percentage of all rheumatoid patients ( $9 \%$ in this series) are likely to be at risk of cervical myelopathy in the first 10 years of disease. It is possible to identify on a clinical basis a group of patients at particular risk of developing severe progressive joint disease. In our study middle-aged females who are strongly seropositive and who develop 'definite' or 'classical' disease (ARA criteria) in the first year of disease and progress to poor functional capacity constitute the high risk group. Cervical subluxations are usually clinically 'silent', at least initially, and radiographs of the cervical spine should be performed in the patients of the high-risk group. Our results suggest that at least one radiograph of the cervical spine should be taken in the first 2 years of disease, especially if peripheral erosive disease is present. More than half the patients with peripheral erosive disease will have some rheumatoid neck changes. If the changes in the peripheral radiographs deteriorate or cervical subluxation is present, then further annual radiographs can be taken to assess progression. 
Cervical myelopathy may develop suddenly after trauma in a patient with an unstable neck. Such patients are liable to fall in awkward positions and are unable to reduce the impact of their fall because of stiffness and limited joint mobility. The onset of myelopathy under these circumstances is a disastrous and often fatal complication. Conversely, a myelopathy may develop slowly over many years, and the treatment of an established case is always difficult. Often cervical decompression and fusion is deferred because of the patient's general disability. We suggest that prophylatic cervical fusion should be more readily considered after following up the small group of rheumatoid patients who develop progressive cervical subluxation in the course of time.

Marks and Sharp ${ }^{12}$ reported a mean delay of 16 years (range 6 to 37 years) from the onset of RA to the clinical detection of cervical myelopathy. In our first report of this prospective study ${ }^{2}$ (mean follow-up $7 \cdot 2$ years) none of our patients had developed myelopathy, supporting the view that neurological signs of this complication do not occur early in the course of the disease and that other factors like disease duration are significant. This report is on the first 15 years only of a detailed study of RA (mean follow-up 9.5 years), and further follow-up will continue. Although we have demonstrated a close relationship between cervical and peripheral $x$-ray changes, other factors such as occupation have independent effects and will be the subject of further study.
We thank Drs A. C. Boyle, S. Mattingly, and D. L. Woolf for permission to study their patients.

The study has been generously supported by the Arthritis and Rheumatism Council.

\section{References}

1 Brook A S, Corbett M. Radiographic changes in early rheumatoid disease. Ann Rheum Dis 1977; 36: 71-3.

2 Winfield J, Cooke D, Brook A S. Corbett M. A prospective study of the radiological changes in the cervical spine in early rheumatoid disease. Ann Rheum Dis 1981; 40: 109-14.

3 Fleming A, Crown J M, Corbett M. Early rheumatoid disease. I. Onset. Ann Rheum Dis 1976; 35: 357-60.

4 Ropes M W, Bennett G A, Cobb S, Jacox R, Jessar R A. The revised ARA criteria for RA. Ann Rheum Dis 1959; 18: 49-53.

5 Steinbrocker O, Traeger C H, Batterman R C. Therapeutic criteria in rheumatoid arthritis. JAMA 1949; 140: 659-62.

6 Lawrence J S. Rheumatism in populations. London: Heinmann, 1977.

7 Bland J H. Rheumatoid arthritis of the cervical spine. J Rheumatol 1974; 1: 319-42.

8 Young A, Brook A S, Corbett M. The clinical assessment of joint inflammatory activity in rheumatoid arthritis related to radiological progression. Rheumatol Rehabil 1980; 19: 14-9.

9 Brook A S, Fleming A, Corbett M. Relationship of radiological changes to clinical outcome in rheumatoid arthritis. Ann Rheum Dis 1977; 39: 274-5.

10 Sharp J, Purser D W, Lawrence J S. Rheumatoid arthritis of the cervical spine in the adult. Ann Rheum Dis 1958; 17: 303-13.

11 Rasker J J, Cosh J A. Radiological study of cervical spine and hand in patients with rheumatoid arthritis of 15 years' duration: an assessment of the effects of corticosteroid treatment. Ann Rheum Dis 1978; 37: 529-35.

12 Marks J S, Sharp J. Rheumatoid cervical myelopathy. $Q J$ Med 1981; 40: 109-14. 